Article

\title{
Supramolecular Structure and Renaturation of a $(1 \rightarrow 3)-\beta$-D-Glucan Compared with Curdlan and Scleroglucan
}

\section{Ana Isabel Puertas ${ }^{1}, M^{a}$ Teresa Dueñas ${ }^{1}$ and Cristina Marieta ${ }^{1,2, *}$}

1 Department of Applied Chemistry, University of the Basque Country, UPV/EHU, $\mathrm{P}^{\mathrm{o}}$ Manuel Lardiazabal, 3, 20018 Donostia-San Sebastián, Spain; E-Mails: anaisabel.puertas@ehu.es (A.I.P.); mariateresa.duenas@ehu.es (M.T.D.)

2 Department of Chemical Ingeniering and Environmental, EUPD, University of the Basque Country, UPV/EHU, Plaza Europa 1, 20018 Donostia-San Sebastián, Spain

* Author to whom correspondence should be addressed; E-Mail: cristina.marieta@ehu.es; Tel.: +34-943-017-190.

Received: 26 July 2014; in revised form: 25 August 2014 / Accepted: 5 September 2014 / Published: 22 September 2014

\begin{abstract}
A ( $1 \rightarrow 3)-\beta$-D-Glucan produced by Lactobacillus suebicus CUPV221 strain was investigated by tapping mode atomic force microscopy (TM-AFM), to compare its supramolecular structure and conformation with two commercial polysaccharides: curdlan and scleroglucan. It was found that the $\beta$-D-Glucan was a $(1 \rightarrow 3)(1 \rightarrow 2)-\beta$-D-Glucan and at room temperature formed three-dimensional networks by entanglements between strands, as does scleroglucan. However, $(1 \rightarrow 3)(1 \rightarrow 2)-\beta$-D-Glucan strands seemed to be more stiff than those of scleroglucan. It was also observed that curdlan samples deposited from $5 \mathrm{mM} \mathrm{NaOH}$ aqueous solution showed supermolecular assemblies, recognized in the literature as micelles, which are controlled by hydrophobic hydration. The $(1 \rightarrow 3)(1 \rightarrow 2)-\beta$-D-Glucan in alkaline aqueous solutions produced different supramolecular structures depending on $\mathrm{pH}$, and at $0.4 \mathrm{M} \mathrm{NaOH}$ ( $\mathrm{pH}$ 13.16), denaturation took place. After neutralizing the alkaline solution with $\mathrm{HCl}$, the formation of short linear, circular, and hairpin structures was observed.
\end{abstract}

Keywords: $(1 \rightarrow 3)-\beta-D-G l u c a n s$; curdlan; scleroglucan; atomic force microscope 


\section{Introduction}

$(1 \rightarrow 3)-\beta$-D-Glucans from several bacteria and fungi constitute a group of natural polysaccharides with a main chain of $(1 \rightarrow 3)$-linked $\beta$-glucopyranosyl units. They can be linear or branched with either $(1 \rightarrow 6)$ or $(1 \rightarrow 2)$-linked side chains of varying length and distribution. In plants, $(1 \rightarrow 3)$ - $\beta$-D-Glucans exhibit a linear structure with mixed $\beta-(1 \rightarrow 3)$ and $\beta-(1 \rightarrow 4)$ glycosidic bonds [1]. These $\beta$-glucans have applications in food, pharmaceutical and other industries as emulsifiers, stabilizers, binders, gelling agents, lubricants, and thickening agents [2]. However, a better understanding of the structure-function relationship of these biopolymers remains a challenge to further improve applications and to better satisfy the consumer demand for appealing, tasty and even healthier products [3]. Moreover, these polysaccharides have been extensively studied in the last decade because they have potential as biological response modifiers. Numerous publications describe their biological activities and therapeutic uses [4], such as antitumor, antibacterial, antiviral, and anticoagulatory effects [5]. The biological activity of these macromolecules is strongly dependent upon chemical as well as physical properties such as conformation or structure, which in turn depend on the environmental conditions [4].

With respect to the industrial uses of $(1 \rightarrow 3)-\beta$-D-Glucans, the strictly linear polysaccharide curdlan is the most studied [6], due to its unique rheological and thermal gelling properties [7] and its ability to form an elastic gel with strong bioactivity [8]. Additionally, its use as an inert dietary fiber is approved in Japan and is registered in the United States as a food additive. Although curdlan has been widely studied [7-9], it is possible to contribute to the characterization of this biomacromolecule since unusual and conflicting results can still be found, especially related to its structural analysis [10]. Another $(1 \rightarrow 3)-\beta$-D-Glucan of industrial importance is scleroglucan, which consists of a main linear chain of $(1 \rightarrow 3)-\beta$-D-Glucopyranosyl units, with one $\beta$-D-Glucopyranosyl unit $(1 \rightarrow 6)$ linked to every third unit of the main chain [11]. The most important industrial application of this polymer is in oil recovery and drilling [2], and its use as an antitumor, antiviral, and antimicrobial compound is being investigated [12].

A new class of $(1 \rightarrow 3)-\beta$-D-Glucans synthesized by some lactic acid bacteria (LAB) has been described. These polysaccharides have a primary structure consisting of a trisaccharide-repeating unit with two $(1 \rightarrow 3)-\beta$ linked glucose residues in the main chain, one of which is substituted in position 2 by a terminal glucose. Their gelling properties have also been reported. Since several LAB species have a "Generally Regarded As Safe" (GRAS) status by the American Food and Drug Association, these $\beta$-glucans could be an alternative to $(1 \rightarrow 3)-\beta$-D-Glucans from non-GRAS microbiota. In addition, it has been shown that the $(1 \rightarrow 3)(1 \rightarrow 2)-\beta$-D-Glucan producing Pediococcus parvulus 2.6 exhibits probiotic properties [13] and assays on human ingestion of an oat-based product manufactured with this strain showed a decrease of serum cholesterol levels [14].

The objective of the work has been to study the supramolecular structure and renaturation of the 2 -branched $(1 \rightarrow 3)-\beta$-D-Glucan produced by Lactobacillus suebicus CUPV221, and to compare the results with those exhibited by curdlan and scleroglucan, which are already of commercial interest. 


\section{Experimental Section}

The polymers used in the present study were as follows: curdlan C7821, which was supplied by Sigma-Aldrich, scleroglucan Actigum ${ }^{\mathrm{TM}}$ CS 11, kindly supplied by Cargill S.L.U. (Martorell, Spain), both polysaccharides were used without any treatment, and a $(1 \rightarrow 3)(1 \rightarrow 2)-\beta$-D-Glucan a exopolysaccharide from a Lactobacillus suebicus CUPV221, a bacterium isolated from a ropy Basque cider. Strain was routinely cultured at $28^{\circ} \mathrm{C}$ in Man Rogosa Sharpe (MRS) broth (Pronadisa, Madrid, Spain), and stored in this medium with glycerol at $20 \%(\mathrm{v} / \mathrm{v})$. For exopolysaccharide production a semidefined (SMD) broth was used without yeast extract, beef extract or peptone, as these ingredients interfere with the $\beta$-glucan purification; it contained (in grams per liter of distilled water): glucose 20, casamino acids (Difco) 10, sodium acetate 5, Bacto yeast nitrogen base (BYNB) (Difco) 6.7, $\mathrm{K}_{2} \mathrm{HPO}_{4} 1$, $\mathrm{KH}_{2} \mathrm{PO}_{4} 1, \mathrm{Mg}_{2} \mathrm{SO}_{4} \cdot 7 \mathrm{H}_{2} \mathrm{O} 0.2, \mathrm{MnSO}_{4} \cdot 4 \mathrm{H}_{2} \mathrm{O} 0.1, \mathrm{KCl} 0.45$, di-ammonium citrate 3.5, Tween 801 , adenine, uracil, thymine and guanine 0.005 . Both glucose and BYNB were sterilized by filtering through a $0.22-\mu \mathrm{m}$-pore size Millex-GS filter unit (Millipore, Bedford, TX, USA) and added to the other, autoclaved ingredients. The $\mathrm{pH}$ of the SMD medium was adjusted to 4.8 prior to sterilization. The clear supernatant obtained by centrifugation for $30 \mathrm{~min}$ at 16,000 $\mathrm{g}$ was collected. Crude EPS was precipitated from the supernatant by addition of three volumes of cold ethanol, followed by storage overnight at $4{ }^{\circ} \mathrm{C}$. The polysaccharide was purified by precipitation with ethanol three times and the final precipitate was resuspended in distilled water, dialyzed (Mw cut-off 12,000-14,000 Da) against distilled water for $48 \mathrm{~h}$ with water replacement twice a day, and finally lyophilized.

The supramolecular structure and conformation of the polysaccharides were studied by tapping mode atomic force microscopy (TM-AFM) performed on a scanning probe microscope (SPM) (Nanoscope IVa, Multimode ${ }^{\mathrm{TM}}$ from Digital Instruments) operating in tapping mode (TM-AFM) at room temperature. Samples were placed on top of a "J" piezoelectric scanner, the maximum $x y$ imaging range of which was $\sim 100 \mu \mathrm{m}$, and scanned at a frequency of $0.2-1 \mathrm{~Hz}$ using the MPP-12100 silicon probes of Veeco. Several specimens were scanned in different regions and similar images were obtained, thus demonstrating the reproducibility of the results. All images are shown without any image processing except in some cases where horizontal leveling and contrast enhancement were used. The diameters of helical units and strands of the polysaccharide were measured with Digital Instruments Nanoscope IV Software version 5.12r5.

The drop deposition method was used to prepare the TM-AFM samples, thus dissolutions of the biopolymers were required. Scleroglucan and the $\beta$-D-Glucan from a Lactobacillus suebicus were dissolved in water at $10 \mathrm{mg} / \mathrm{L}$, and curdlan, which is not soluble in pure water, was dissolved in $5 \mathrm{mM} \mathrm{NaOH}$ aqueous solution, at a concentration of $10 \mathrm{mg} / \mathrm{L} .0 .5 \mu \mathrm{L}$ of the solutions were pipetted onto cleaved sheets of mica. The samples were air-dried in a desiccator at atmosphere pressure for $24 \mathrm{~h}$.

We found that at $0.4 \mathrm{M} \mathrm{NaOH}(\mathrm{pH} 13.16)$ the strand separation of triplexes took place (denaturation). Some authors have documented the ability of the triple helices to spontaneously re-form upon restoration of the thermodynamic conditions (renaturation) [4]. To study the renaturation of the $(1 \rightarrow 3)-\beta$-D-Glucan, a $1-\mathrm{mg} / \mathrm{L}$ solution in $0.4 \mathrm{M} \mathrm{NaOH}$ was neutralized with $\mathrm{HCl}$ and the salt was removed by dialysis. Then, the previous described drop deposition method was used to prepare the samples for TM-AFM. 


\section{Results and Discussion}

$(1 \rightarrow 3)-\beta-D-G l u c a n$ is produced by a lactic acid bacterium, Lactobacillus suebicus CUPV221 [15]. This biopolymer is a main linear chain of $(1 \rightarrow 3)-\beta$-D-Glucopyranosyl units, with one $(1 \rightarrow 2)-\beta$-D-Glucopyranosyl pendant unit attached to every two units of the main chain, as shown in Figure $1[16]$.

Figure 1. Repeat unit of the polysaccharide produced by Lactobacillus suebicus CUPV221.

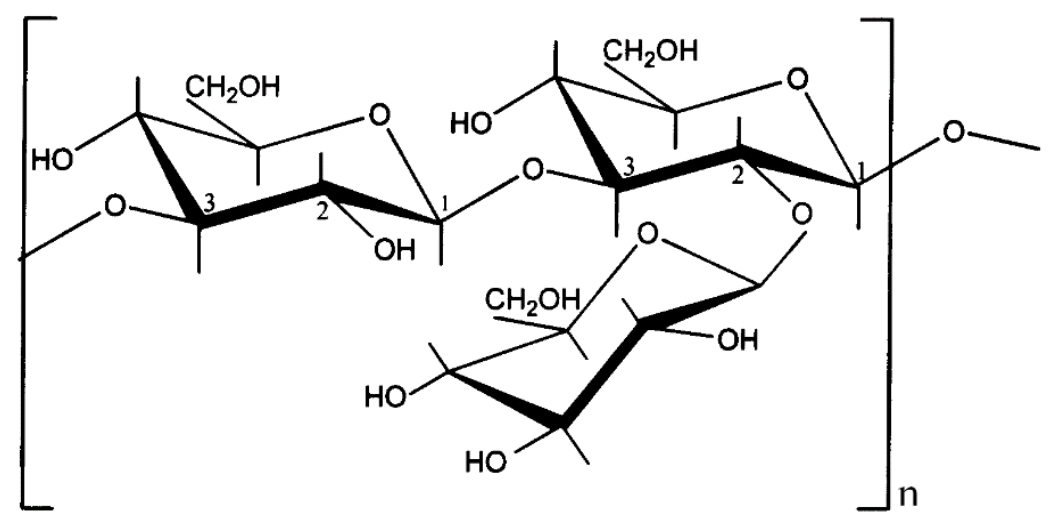

At room temperature, this $(1 \rightarrow 3)(1 \rightarrow 2)-\beta$-D-Glucan forms a gel network structure, that we probed by TM-AFM, Figure $2 \mathrm{a}$.

Figure 2. TM-AFM height images of: (a) $(1 \rightarrow 3)(1 \rightarrow 2)-\beta-D-G l u c a n ;(\mathbf{b})$ scleroglucan. Images were obtained by depositing the glucans from $10 \mathrm{mg} / \mathrm{L}$ aqueous solutions onto mica and scanning in air. Height images of $1.5 \mu \mathrm{m} \times 1.5 \mu \mathrm{m}$.

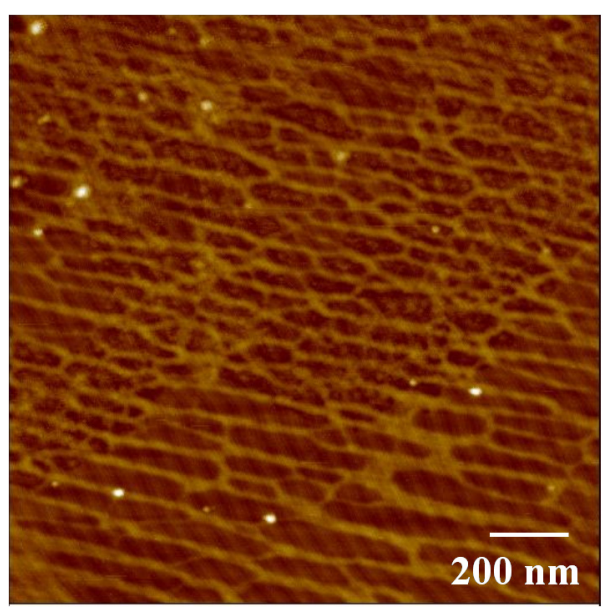

(a)

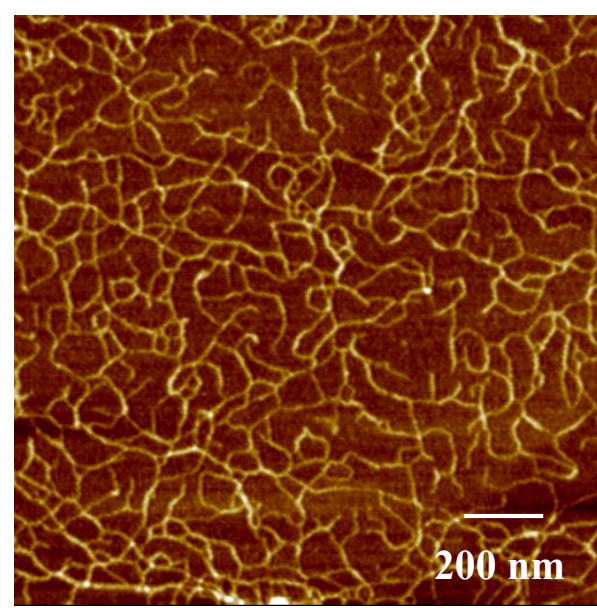

(b)

Additionally, we previously performed a study of an identical polysaccharide, a branched 2 -substituted $(1 \rightarrow 3)-\beta$-D-Glucan, produced by $P$. parvulus 2.6 , and the existence of a physical network in water solution at room temperature was proved by rheological measurements [17]. Furthermore, two facts demonstrated that it was a low-set gel or weak gel: (i) viscosity decreased above a certain shear rate, and (ii) the viscous modulus $\left(G^{\prime \prime}\right)$ was lower than the elastic modulus $\left(G^{\prime}\right)$, with a very slight dependence on frequency, behavior associated with weak gels [18]. Similar weak gel behavior has been 
described for scleroglucan [19]. Figure $2 \mathrm{~b}$ depicts a TM-AFM image of a sample from a $10 \mathrm{mg} / \mathrm{L}$ aqueous solution of scleroglucan. The image shows a three-dimensional network formed by entanglements between strands like that of the $(1 \rightarrow 3)(1 \rightarrow 2)-\beta$-D-Glucan and scleroglucan (Figure $2 a)$.

Due to the rod like character of the $(1 \rightarrow 3)(1 \rightarrow 2)-\beta$-D-Glucan strands and their unwinding observed at $13.16 \mathrm{pH}$, a helical conformation was assigned [20]. The conformation of scleroglucan is also widely accepted as a rod-like triple helix [21,22]. However, in view of the TM-AFM images, Figure 2, and keeping in mind the repeating units of both biopolymers, it can be concluded that the strands of $(1 \rightarrow 3)(1 \rightarrow 2)-\beta$-D-Glucan are more stiff than those of scleroglucan.

In its solid-state, commercial curdlan is insoluble in water at room temperature. Therefore, to carry out the study by AFM it was necessary to prepare a solution with a suitable solvent. Due to the ionization of hydrogen bonds, curdlan dissolves easily in dilute alkalinesolutions. When this solvent is used to carry out morphological and structural studies, curdlan shows different morphologies depending on $\mathrm{pH}$, ranging from endless microfibrils to spindle-shaped fibrils of various lengths [23], or even thin lamellar hexagonal crystals [24], and the conformation varies from triple or single helix to random coil [25]. When a weak alkaline solution is used to prepare the AFM samples, the curdlan structure could almost be maintained [8]. Thus, $5 \mathrm{mM} \mathrm{NaOH}$ aqueous solution, $\mathrm{pH} 11.75$, was chosen to develop the present study. Figure 3 shows the morphology of curdlan after the evaporation of the solvent at room temperature. A height image (left) reveals a fairly rough morphology with a high density of spike-like growth features. Observing the phase image (right), a homogenous color of the image is seen. This means that more curdlan exists between the spindle-shaped joined microfibrils forming the spike-like features. When a higher magnification of this area was made, $2.5 \mu \mathrm{m} \times 2.5 \mu \mathrm{m}$ scan, an entanglement of fibrils composed of laterally associated strands were observed (Figure $3 b$ ).

Figure 3. TM-AFM height (left) and phase (right) images of curdlan. Images were obtained by depositing the glucan from a $10 \mathrm{mg} / \mathrm{L} 5 \mathrm{mM} \mathrm{NaOH}$ aqueous solution, $\mathrm{pH} 11.75$, onto mica and scanned in air: (a) images of $10 \mu \mathrm{m} \times 10 \mu \mathrm{m}$; (b) images of $2.5 \mu \mathrm{m} \times 2.5 \mu \mathrm{m}$.

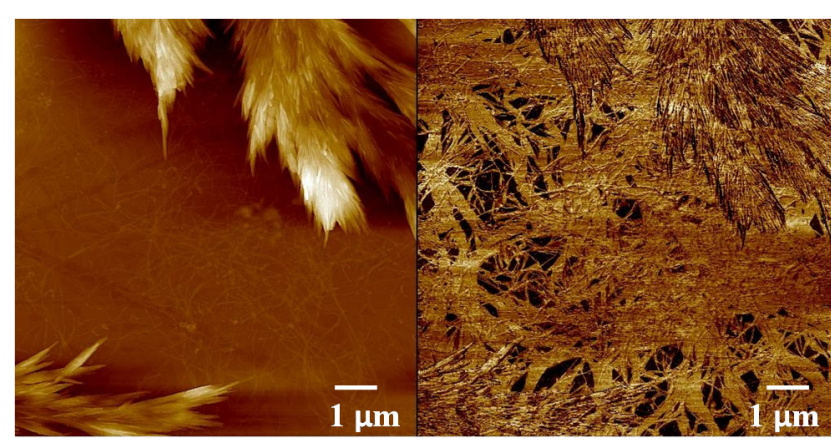

(a)

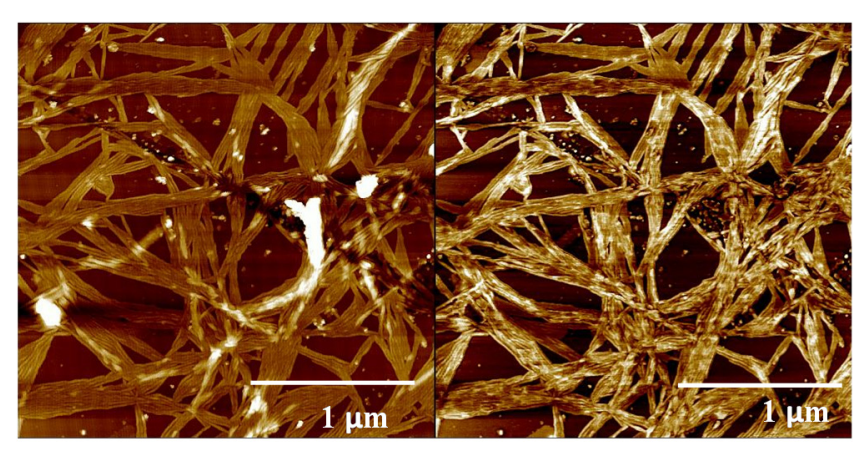

(b)

Trace analyses were also carried out to obtain quantitative information from AFM images. The diameter of the strands for curdlan was about $1.6 \mathrm{~nm}$, slightly larger than that reported for the triple strand thickness by X-ray fiber diffraction, $1.56 \mathrm{~nm}[8,26]$. Moreover, this discrepancy between values by AFM and those determined by other techniques has already observed by other authors, and they propose that the dissimilarity might result from the fact that the molecules were distorted by desiccation or by interaction with the mica substrate, or that the molecules were partially embedded in a layer of 
water adhering to the mica surface [27]. The diameter of the strands of the $(1 \rightarrow 3)(1 \rightarrow 2)-\beta$-D-Glucan was about $1.8 \mathrm{~nm}$ [20], similar to that found in the literature for scleroglucan [28].

The structure of the $(1 \rightarrow 3)(1 \rightarrow 2)-\beta$-D-Glucans in units of helical conformation is an important basis for their functional properties. Nevertheless, this helical conformation is not stable at high $\mathrm{pH}$ or by increasing the temperature above the helix melting temperature. Thus, breaking of intermolecular hydrogen bonds takes place and the multi-helical units dissociate into single stranded random coils (denaturation) [4]. However, the triple helix can be reformed by neutralizing. Different structures have been mentioned in literature for renatured species: linear, circular, hairpin, multichain (aggregated) structures, etc. $[4,17]$. The aim of the present work was to study the conformational transitions during the denaturation-renaturation for the $(1 \rightarrow 3)(1 \rightarrow 2)-\beta$-D-Glucan and to compare its behaviour with the scleroglucan behaviour from previous works found in the literature $[4,29]$. The denaturation of the $(1 \rightarrow 3)(1 \rightarrow 2)-\beta$-D-Glucan took place at $0.4 \mathrm{M} \mathrm{NaOH}$ aqueous solution $(\mathrm{pH}$ 13.16) and after the neutralization TM-AFM images were taken.

As can be seen in Figure 4, a mixture of linear, circular and hairpin structures in addition to larger clusters with less well-defined morphology were found. Similar behavior was observed for scleroglucan [4] and schizophyllan [30]. However, it should be mentioned that, for the $(1 \rightarrow 3)(1 \rightarrow 2)-\beta$-D-Glucan, a great dispersion structure sizes was observed (scales are shown below each image), small linear and circular structures (Figure 4a) coexisted with strands of $5 \mu \mathrm{m}$ of length (Figure $4 \mathrm{~b}$ ) and small circular structures (Figure 4a) with rod-like linear strands (Figure 4c). This behavior could be attributed to the more stiff character of the $(1 \rightarrow 3)(1 \rightarrow 2)-\beta$-D-Glucan biomacromolecules. The strand separation of triplexes was originally considered to be irreversible [4], but later extensive analysis of many micrographs using scleroglucan samples in five different ranges of molecular weight suggested that the reconstituted molecules, both rods and rings, are substantially triple helical [31].

Figure 4. TM-AFM height images of renaturated $(1 \rightarrow 3)(1 \rightarrow 2)-\beta$-D-Glucan. Images were obtained by depositing the glucan from the $(1 \rightarrow 3)(1 \rightarrow 2)-\beta$-D-Glucan neutralized solution.

(a) Images of $10 \mu \mathrm{m} \times 10 \mu \mathrm{m}$; (b) image of $7.11 \mu \mathrm{m} \times 7.11 \mu \mathrm{m}$; (c) image of $1.5 \mu \mathrm{m} \times 1.5 \mu \mathrm{m}$.

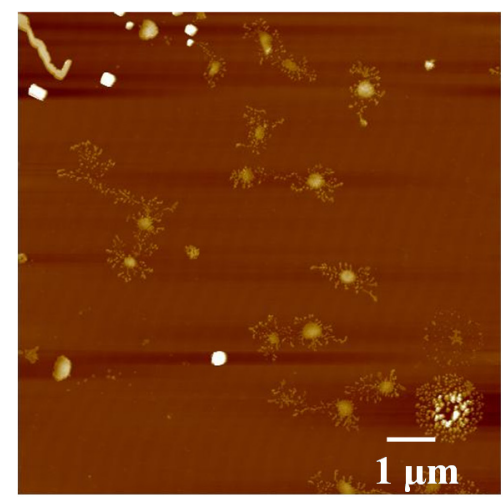

(a)

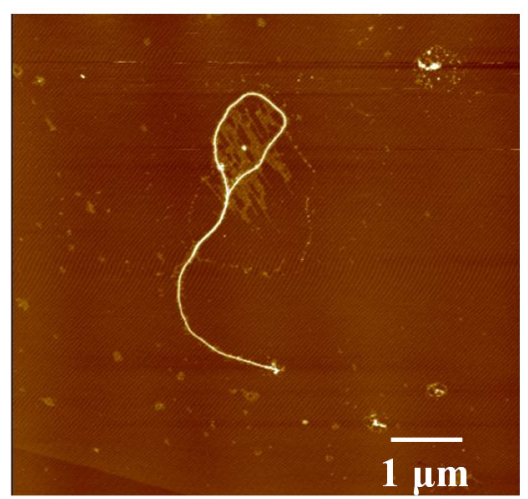

(b)

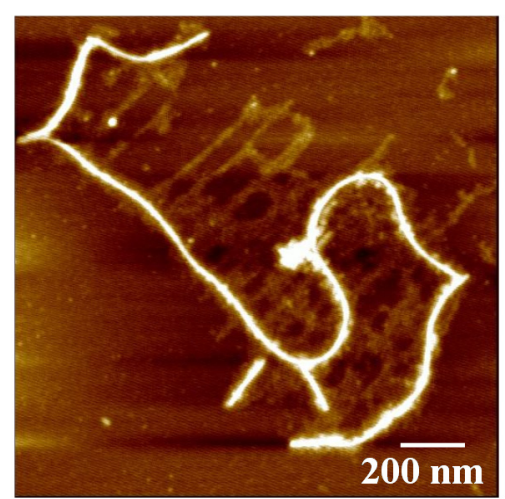

(c)

\section{Conclusions}

We have shown that the $(1 \rightarrow 3)(1 \rightarrow 2)-\beta$-D-Glucan from Lactobacillus suebicus CUPV221 forms three-dimensional network structures by entanglements between strands, as occurs with scleroglucan. 
Due to the macromolecular structure and also possibly to the direction of the helical arrangement, $(1 \rightarrow 3)(1 \rightarrow 2)-\beta$-D-Glucan strands are more stiff than those of scleroglucan. Curdlan samples deposited from $5 \mathrm{mM} \mathrm{NaOH}$ aqueous solution showed supermolecular assemblies, which are recognized in the literature as micelles.

$(1 \rightarrow 3)(1 \rightarrow 2)-\beta$-D-Glucan after exposure to a denaturation-renaturation cycle forms various molecular topologies: linear, circular and hairpin structures in addition to larger clusters with less well-defined morphology.

\section{Acknowledgments}

This study was supported by grants AGL2009-12998-C03 and AGL2012-40084 from the Spanish Ministry of Science and Innovation, and IT335-10 from the Basque Government. Ana Isabel Puertas acknowledges the "Gobierno Vasco, Dpto. Agricultura, Pesca y Alimentación” for the fellowship.

\section{Author Contributions}

The manuscript was finalized through contributions from all authors, and all authors also approved the final manuscript.

\section{Conflicts of Interest}

The authors declare no conflict of interest.

\section{References}

1. Laroche, C.; Michaud, P. New developments and prospective applications for $\beta-(1,3)$ glucans. Recent Pat. Biotechnol. 2007, 1, 59-73.

2. Survase, S.A.; Saudagar, P.S.; Bajaj, I.B.; Singhal, R.S. Scleroglucan: Fermentative production, downstream processing and applications. Food Technol. Biotecnol. 2007, 45, 107-118.

3. Duboc, P.; Mollet, B. Applications of exopolysaccharides. Int. Dairy J. 2001, 11, 759-768.

4. Sletmoen, M.; Stokke, B.T. Higher order structure of $(1,3)-\beta$-D-glucans and its influence on their biological activities and complexation abilities. Biopolymers 2008, 89, 310-320.

5. Gawronski, M.; Park, J.T.; Magee, A.S.; Conrad, H. Microfibrillar structure of PGG-glucan in aqueous solution as Triple-helix aggregates by small angle x-ray scattering. Biopolymers 1999, 50, 569-578.

6. Harada, T.; Masada, M.; Fujimori, K.; Maeda, I. Production of a firm, resilient gel-forming polysaccharide by a mutant of Alcaligenes faecalis var. myxogenes 10C3. Agric. Biol. Chem. 1966, 30, 196-198.

7. McIntosh, M.; Stone, B.A.; Stanisich, V.A. Curdlan and other bacterial $(1 \rightarrow 3)-\beta$-D-glucans. Appl. Microbiol. Biotechnol. 2005, 68, 163-173.

8. Jin, Y.; Zhang, H.; Yin, Y.; Nishinari, K. Conformation of curdlan as observed by tapping mode atomic force microscopy. Colloid Polym. Sci. 2006, 284, 1371-1377. 
9. Sun, Y.; Liu, Y.; Li, Y.; Lv, M.; Li, P.; Xu, H.; Wang, L. Preparation and characterization of novel curdlan/chitosan blending menbranes for antibacterial applications. Carbohydr. Polym. 2011, 84, 952-959.

10. Zhang, H.; Huang, L.; Nishinari, K.; Watase, M.; Konno, A. Thermal measurements of curdlan in aqueous suspension during gelation. Food Hydrocolloids 2000, 14, 121-124.

11. Vincendon, M. Scleroglucan derivatives: Aromatic carbamates. J. Polym. Sci. Part A Polym. Chem. 1999, 37, 3187-3192.

12. Kim, H.I.; Kim, J.H.; Yang, S.B.; Hong, S.G.; Lee, S.A.; Hwang, S.J.; Shin, K.S.; Suh, H.J.; Park, M.H. A polysaccharide extracted from rice bran fermented with Lentinus edodes enhaces natural killer cell activity and exhibits anticancer effects. J. Med. Food 2007, 10, 25-31.

13. Fernández de Palencia, P.; Werning, M.L.; Sierra-Filardi, E.; Dueñas, M.T.; Irastorza, A.; Corbí, A.L.; López, P. Probiotic properties of the 2 -substituted $(1,3)-\beta$-D-glucan-producing bacterium Pediococcus parvulus 2.6. Appl. Environ. Microbiol. 2009, 75, 4887-4891.

14. Mårtensson, O.; Biörklund, M.; Lambo, A.M.; Dueñas-Chasco, M.T.; Irastorza, A.; Holst, O.; Norin, E.; Walling, G.; Öste, R.; Önning, G. Fermented ropy, oat-based products reduce cholesterol levels and stimulate the bifidobacteria flora in humans. Nutr. Res. 2005, 25, 429-442.

15. Garai-Ibabe, G.; Dueñas, M.T.; Irastorza, A.; Sierra-Filardi, E.; Werning, M.L.; López, P.; Corbí, A.L.; Fernández de Palencia, P. Naturally occurring 2-substituted (1,3)- $\beta$-D-glucan producing Lactobacillus suebicus and Pediococcus parvulus strains with potential utility in the production of functional foods. Bioresour. Technol. 2010, 101, 9254-9263.

16. Ibarburu, I. Exopolysaccharide-producing lactic acid bacteria: Structural analysis of polysaccharides and molecular detection of $\beta-(1 \rightarrow 3)(1 \rightarrow 2)$-D-glucan producer strains. Ph.D. Thesis, University of the Basque Country (UPV/EHU), Donostia-San Sebastián, Spain, 2009.

17. Velasco, S.; Irastorza, A.; Dueñas, M.; Santamaría, A.; Muñoz, M.E. Chemical and rheological properties of the $\beta$-glucan produced by Pediococcus parvulus 2.6. J. Agric. Food Chem. 2009, 57, 1827-1834.

18. Guenet, J.M. Thermorevesible Gelation of Polymers and Biopolymers; Academic Press Inc.: San Diego, CA, USA, 1992.

19. Grassi, M.; Lapasin, R.; Pricl, S. A study of the rheological behavior of scleroglucan weak gel systems. Carbohydr. Polym. 1995, 29, 169-181.

20. Marieta, C.; Ibarburu, I.; Dueñas, M.; Irastorza, A. Supramolecular structure and conformation of a from a ropy strain of Lactobacillus suebicus as observed by tapping mode atomic force microscopy. J. Agric. Food Chem. 2009, 57, 6183-6188.

21. Yanaki, T.; Norisuye, T. Triple helix and random coil scleroglucan in dilute solution. Polym. J. 1983, 15, 389-396.

22. Harada, T.; Misaki, A.; Saito, H. Curdlan: A bacterial-gel-forming $\beta-1 \rightarrow 3$ glucan. Arch. Biochem. Biophys. 1968, 124, 292-298.

23. Harada, T.; Harada, A. Gel Formation and Ultrastructure in Food Polysaccharides. In Polysaccharide Association Structures in Food; CRC Press: Boca Raton, FL, USA, 1998; pp. 37-40.

24. Pelosi, P.; Bulone, V.; Heux, L. Polymorphism of curdlan and $(1 \rightarrow 3)-\beta$-D-glucans synthesized in vitro: A13C CP-MAS and X-Ray diffraction analysis. Carbohydr. Polym. 2006, 66, 199-207. 
25. Zhang, L.; Wang, C.; Cui, S.; Wang, Z.; Zhang, X. Single-molecules force spectroscopy on curdlan: Unwinding helical structures and random coils. Nanoletters 2003, 3, 1119-1124.

26. Nishinari, K. Rheological and DSC study of sol-gel transition in aqueous dispersions of industrially important polymers and colloids. Colloid Polym. Sci. 1997, 275, 1093-1107.

27. McIntire, T.M.; Brant, D.A. Imaging of individual biopolymers and supramolecular assemblies using noncontact atomic force microscopy. Biopolymers 1997, 42, 133-146.

28. McIntire, T.M.; Penner, R.M.; Brant, D.A. Observations of a circular, triple-helical polysaccharide using noncontact atomic force microscopy. Macromolecules 1995, 28, 6375-6377.

29. Vuppu, A.; Garcia, A.A.; Vernia, C. Tapping mode atomic force microscopy of scleroglucan networks. Biopolymers 1997, 42, 89-100.

30. Stokke, B.T.; Elgsaeter, A.; Brant, D.A.; Kuge, T.; Kitamura, S. Macromolecular cyclization of $(1 \rightarrow 6)$-branched- $(1 \rightarrow 3)$ - $\beta$-D-glucans observed after denaturation-renaturation of the triple-helical structure. Biopolymers 1993, 33, 193-198.

31. Brant, D.A. Versatile polysaccharides: Ropes, rings, and rods. AIM Mag. 2005, 60, 40-49.

(C) 2014 by the authors; licensee MDPI, Basel, Switzerland. This article is an open access article distributed under the terms and conditions of the Creative Commons Attribution license (http://creativecommons.org/licenses/by/3.0/). 